\title{
Síntesis y Caracterización de Adhesivos Reposicionables de Poliuretano Dispersos en Agua, Obtenidos a partir de Aceite de Castor Maleinizado
}

\author{
Paula Mazo, Lysa López, David Restrepo, Luis Rios \\ Departamento de Engenharia Química, Universidad de Antioquia
}

Resumen: El presente trabajo se centró en la producción de dispersiones acuosas de poliuretano empleando el método de emulsificación por inversión de fases. Para la síntesis fueron empleados dos polioles: uno comercial tipo poliéster y el otro es aceite de castor maleinizado (MACO); dos diisocianatos, uno aromático Toluen diisocianato (TDI) y otro alifático Hexametilen diisocianato (HDI), siendo poco comunes para esta aplicación. Como emulsificante interno se empleó ácido cítrico el cual es muy económico y de fácil adquisición. Como factores se evaluaron la cantidad de ácido cítrico y la relación molar de grupos isocianato a hidroxilos $(\mathrm{NCO} / \mathrm{OH})$, generando un diseño de experimentos factorial $3^{2}$ y minimizando el contenido de sólidos sedimentados. Mediante la metodología de superficie de respuesta, se obtuvo una ecuación polinomial cuadrática por un análisis de regresión múltiple. Los poliuretanos formados fueron evaluados en cuanto a sus propiedades adhesivas y mecánicas (pegajosidad tack, pegajosidad en cinta loop tack, cohesión hold, pelado peel), lo que permitió clasificar el adhesivo como reposicionable, para emplearlo en etiquetas.

Palabras-clave: Adhesivos reposicionables, poliuretano, dispersiones, aceite de castor.

\section{Synthesis and Characterization of Pressure-Sensitive Adhesives of Waterborne Polyurethane from Maleinizated Castor Oil}

\begin{abstract}
This work focused on the production of aqueous dispersions of polyurethane using the emulsification method by phase inversion. For the synthesis two polyols were employed, viz. a commercial polyester and Maleinizated Castor Oil (MACO), with two diisocyanates: one aromatic, toluene diisocyanate (TDI) and another aliphatic, hexamethylene diisocyanate (HDI), which are not common for this application. Citric acid was used as internal emulsifier which is inexpensive and readily available. The parameters were the amount of citric acid and the mole ratio of isocyanate groups to hydroxyl $(\mathrm{NCO} / \mathrm{OH})$, generating a $3^{2}$ factorial design, with minimization of the sedimented solids. The surface methodology was used to obtain a quadratic polynomial equation by multiple regression analysis. Polyurethanes synthesized were evaluated for their mechanical and adhesive properties (tack, loop tack, hold, peel). These measurements allowed classifying the polyurethanes synthesized as repositionable adhesives, for use in labels.
\end{abstract}

Keywords: Repositionable adhesives, polyurethane, dispersions, castor oil.

\section{Introducción}

Actualmente se está propendiendo a la búsqueda de materas primas alternativas renovables y a la síntesis de polímeros más benignos desde el punto de vista ambiental. En este contexto, es de interés la obtención de materiales poliméricos biodegradables provenientes de aceites naturales ${ }^{[1,2]}$.

Los poliuretanos se obtienen comúnmente empleando dioles de alto peso molecular derivados del petróleo. Con la crisis energética y los problemas asociados al desabastecimiento de esta materia prima se han comenzado a emplear polioles de aceites vegetales como fuentes alternativas, tales como el castor y soja modificada, con la ventaja adicional de bajar el impacto ambiental de los procesos de síntesis ${ }^{[3]}$.

En el campo de las dispersiones acuosas poliméricas una de la aplicaciones más importante está relacionada con la industria de los adhesivos, evidenciada en las grandes cifras consumidas tanto en Europa como en Norte América, que para el año 1996 fueron de 1.5 y 2.3 millones de toneladas, respectivamente. En ese mismo año el mercado mundial de los adhesivos alcanzó una cifra de 21 billones de dólares ${ }^{[4]}$, consecuente con esto se han llevado a cabo un considerable número de investigaciones con el fin de mejorar los resultados obtenidos sobre los adhesivos.

Los adhesivos más empleados a nivel industrial se basan en dispersiones acuosas acrílicas las cuales son

Autor para correspondência: Paula Mazo, Departamento de Ingeniería Química, Universidad de Antioquia, Av Calle 67 № 53-108 Medellín, Colombia. 
petroquímicas, para tratar de sustituirlas se pretende en este trabajo utilizar poliuretanos, los cuales pueden ser formulados para diversas aplicaciones, una de ellas son los adhesivos reposicionables o sensibles a la presión (PSA) empleados en cintas adhesivas.

Entre las investigaciones que se han llevado a cabo para el desarrollo de las dispersiones acuosas de poliuretano (PUD’s), Jang y colaboradores ${ }^{[5]}$, emplearon el método de la acetona, en el cual una solución de poliuretano ionomérico se prepara en una gran cantidad de acetona, la solución se mezcla con agua y se remueve el solvente orgánico por destilación.

El estudio de la incorporación de grupos iónicos en las cadenas de poliuretano comenzó en 1962 en Farbenbriken Bayer Leverkusen (ahora Bayer AG). Los grupos iónicos pueden ser catiónicos, aniónicos o zwitteriónicos, y los contraiones pueden variar ampliamente. Los sitios iónicos pueden estar localizados dentro del esqueleto de cadena (grupos amonio cuaternarios) o pendientes de ella (grupos sulfonato o carboxilato $)^{[6]}$.

El ácido dimetilol propiónico(DMPA) ha sido el reactivo más empleado para la síntesis de PUD’s, confiriéndole gran estabilidad y fácil reactividad, sin embargo este reactivo es de difícil consecución y es muy costoso, lo que encarece aún más los adhesivos.

El uso de materias primas renovables como es el aceite de castor, permite obtener un producto competitivo con los petroquímicos a nivel comercial, de durabilidad y con minimización del impacto ambiental al ser dispersados en agua y biodegradables ${ }^{[7-9]}$.

Con este trabajo se fomenta la obtención de productos renovables, una nueva aplicación para las dispersiones poliuretánicas, con propiedades óptimas para su uso en etiquetas y se fomenta el desarrollo social y agro-industrial en el país.

\section{Experimental}

\section{Reactivos}

Poliol poliéster FL-60 de Swing $\left(\mathrm{OHv}=56 \mathrm{mgKOH} \cdot \mathrm{g}^{-1}\right.$ muestra $)$, Aceite de castor grado USP $\left(\mathrm{OHv}=159.51 \mathrm{mgKOH} . \mathrm{g}^{-1}\right.$ muestra), anhídrido maléico grado comercial (Merk), 2,4 Toluen diisocianato (TDI) grado comercial (Merk), Hexametilen diisocianato grado comercial (Merk), agua corriente sin ningún tratamiento, Trietilamina grado comercial (Merk), Octoato de estaño grado comercial (Tego), tolueno grado analítico (Merk).

\section{Preparación Aceite de Castor Maleinizado (MACO)}

$\mathrm{El}$ aceite de castor maleinizado fue preparado de acuerdo a trabajos anteriores ${ }^{[10]}$, el anhídrido maléico y el aceite de castor fueron adicionados con una relación molar 1:1 en un reactor de $250 \mathrm{~mL}$, se mantuvo la reacción mediante agitación por 4 horas a $90{ }^{\circ} \mathrm{C}$. El aceite obtenido presenta un valor de hidroxilos de $100 \mathrm{mgKOH} . \mathrm{g}^{-1}$ muestra y un valor ácido de $57 \mathrm{mgKOH} . \mathrm{g}^{-1}$ muestra de acuerdo con las normas ASTM D4274 - 05 y ASTM D4662 - 03, respectivamente.

\section{Preparación PUD's}

En el proceso de obtención se realiza en cuatro pasos:

1. Elaboración del prepolímero con grupos isocianato libres mediante la reacción del diisocianato con el poliol y el ácido cítrico, en acetona como solvente, en un reactor de vidrio tipo Kettle con capacidad de $250 \mathrm{~mL}$, agitador mecánico, manta de calentamiento con control de temperatura, un condensador y atmósfera inerte (nitrógeno).

2. Neutralización del prepolímero con trietilamina (TEA) (30 minutos).

3. Dispersión del prepolímero neutralizado lentamente en agua (1 hora).

4. Recuperación del solvente orgánico por evaporación.

\section{Diseño experimental}

Se realizó un diseño factorial $3^{2}$ con cuatro repeticiones al centro y se optimiza mediante la metodología de superficie de respuesta. Los variables y niveles se especifican en la Tabla 1 , el diseño experimental en la Tabla 2.

\section{Caracterización de los polioles, prepolímeros y adhesivos}

Valor de hidroxilos (ASTM D4274 - 05), Valor de acidez (ASTM D4662 - 03), \% Isocianato libre (ASTM D5155-01), Viscosidad (ASTM D-1084), Tack (ASTM D-3121), Loop Tack (ASTM D-6195), peel ( ASTM D-1876), contenido sólidos total, espectroscopía IR (Perkin Elmer), Calorimetría diferencial de barrido DSC (TA instrument).

\section{Resultados y Análisis}

\section{Cinética de la reacción para la obtención de los prepolímeros}

La Figura 1 muestra la disminución del \%NCO libre para los sistemas PES FL-60-TDI, PES FL-60-HDI, MACO-TDI, MACO-HDI, experimentos formulados con una relación molar de $\mathrm{NCO}: \mathrm{OH}=1$, considerando la cantidad de hidroxilos y acidez de todos los reactivos, para cada uno de los sistemas, a $90{ }^{\circ} \mathrm{C}$, sin catalizador.

En el caso donde la concentración de grupos isocianato sea igual a los hidroxilos y/o grupos reactivos, la cinética puede ser de segundo orden ${ }^{[11,12]}$, además los valores de $\mathrm{R}^{2}$ para estos sistemas en estudio reafirman esto (Tabla 3). El mecanismo de la catálisis básica de formación de PU's es mostrado en la Figura 2.

Tabla 1. Variables y niveles empleados en la preparación de las dispersiones.

\begin{tabular}{llccc}
\hline \multirow{2}{*}{} & Variables & \multicolumn{3}{c}{ Niveles } \\
\cline { 3 - 5 } & & $\mathbf{- 1}$ & $\mathbf{0}$ & $\mathbf{1}$ \\
\hline 1 & \%Cítrico* & $1 \%$ & $2.5 \%$ & $5 \%$ \\
2 & NCO:OH & 0.9 & 1.1 & 1.3 \\
\hline
\end{tabular}

\%Citrico: el porcentaje de ácido cítrico se refiere al porcentaje en peso respecto a la cantidad de poliol que se empleó en cada ensayo. NCO:OH: relación molar de grupos isocianato a grupos hidroxilos y ácido. 
Tabla 2. Cantidades de reactivos para los ensayos.

\begin{tabular}{|c|c|c|c|c|c|c|c|}
\hline & \multicolumn{7}{|c|}{ Diseño de experimentos } \\
\hline & Ensayo & VBL 1 & VBL 2 & Poliol (g) & TDI (g) & HDI (g) & Cítrico $(\mathrm{g})$ \\
\hline \multirow{9}{*}{$\begin{array}{l}0 \\
0 \\
1 \\
1 \\
1 \\
2 \\
\text { TI } \\
2\end{array}$} & 1 & -1 & -1 & 15 & 1.40 & 1.35 & 0.150 \\
\hline & 2 & 1 & -1 & 15 & 1.89 & 1.82 & 0.750 \\
\hline & 3 & 0 & 1 & 15 & 2.29 & 2.21 & 0.375 \\
\hline & 4 & 1 & 0 & 15 & 2.31 & 2.23 & 0.750 \\
\hline & 5 & 0 & -1 & 15 & 1.59 & 1.53 & 0.375 \\
\hline & 6 & 1 & 1 & 15 & 2.73 & 2.63 & 0.750 \\
\hline & 7 & 0 & 0 & 15 & 1.94 & 1.87 & 0.375 \\
\hline & 8 & -1 & 1 & 15 & 2.02 & 1.95 & 0.150 \\
\hline & 9 & -1 & 0 & 15 & 1.71 & 1.65 & 0.150 \\
\hline \multirow{19}{*}{\begin{tabular}{l}
0 \\
\multirow{Z}{Z}{}
\end{tabular}} & 10 & 0 & 0 & 15 & 1.94 & 1.87 & 0.375 \\
\hline & 11 & 0 & 0 & 15 & 1.94 & 1.87 & 0.375 \\
\hline & 12 & 0 & 0 & 15 & 1.94 & 1.87 & 0.375 \\
\hline & 13 & 0 & 0 & 15 & 1.94 & 1.87 & 0.375 \\
\hline & 14 & 0 & 0 & 15 & 1.94 & 1.87 & 0.375 \\
\hline & 15 & -1 & -1 & 15 & 2.22 & 2.14 & 0.150 \\
\hline & 16 & 1 & -1 & 15 & 2.71 & 2.61 & 0.750 \\
\hline & 17 & 0 & 1 & 15 & 3.47 & 3.35 & 0.375 \\
\hline & 18 & 1 & 0 & 15 & 3.31 & 3.19 & 0.750 \\
\hline & 19 & 0 & -1 & 15 & 2.40 & 2.32 & 0.375 \\
\hline & 20 & 1 & 1 & 15 & 3.91 & 3.77 & 0.750 \\
\hline & 21 & 0 & 0 & 15 & 2.94 & 2.83 & 0.375 \\
\hline & 22 & -1 & 1 & 15 & 3.21 & 3.09 & 0.150 \\
\hline & 23 & -1 & 0 & 15 & 2.71 & 2.61 & 0.150 \\
\hline & 24 & 0 & 0 & 15 & 2.94 & 2.83 & 0.375 \\
\hline & 25 & 0 & 0 & 15 & 2.94 & 2.83 & 0.375 \\
\hline & 26 & 0 & 0 & 15 & 2.94 & 2.83 & 0.375 \\
\hline & 27 & 0 & 0 & 15 & 2.94 & 2.83 & 0.375 \\
\hline & 28 & 0 & 0 & 15 & 2.94 & 2.83 & 0.375 \\
\hline
\end{tabular}

Asumiendo una cinética de segundo orden con respecto al diisocianato tenemos (Ecuacion 1):

$$
-\frac{d C_{N C O}}{d t}=k C_{N C O}^{2}
$$

Integrando se tiene (Ecuacion 2):

$$
\frac{\eta_{N C O}}{C_{N C O 0}\left(1-\eta_{N C O}\right)}=k t
$$

donde, $\mathrm{C}_{\mathrm{NCO}}$ : concentración de grupos $\mathrm{NCO}$ en el tiempo t; $\mathrm{C}_{\mathrm{NCO} 0}$ : concentración de grupos $\mathrm{NCO}$ en el tiempo $\mathrm{t}=0$; $\eta_{\mathrm{NCO}}$ : conversión de grupos isocianato; $\mathrm{k}$ : constante de reacción (L/mol-s).

Graficando la Ecuación 2 se puede obtener las constantes de reacción a $90{ }^{\circ} \mathrm{C}$ (Figura 3 a y b).

El TDI presenta dos grupos isocianato con diferente reactividad, por lo tanto existen dos constantes de velocidad para la reacción sin catalizador (Figura 3a), el HDI tiene dos grupos isocianato de igual reactividad, por lo cual presenta una sola constante de velocidad (Figura 3b), estos se reportan en la Tabla 3.

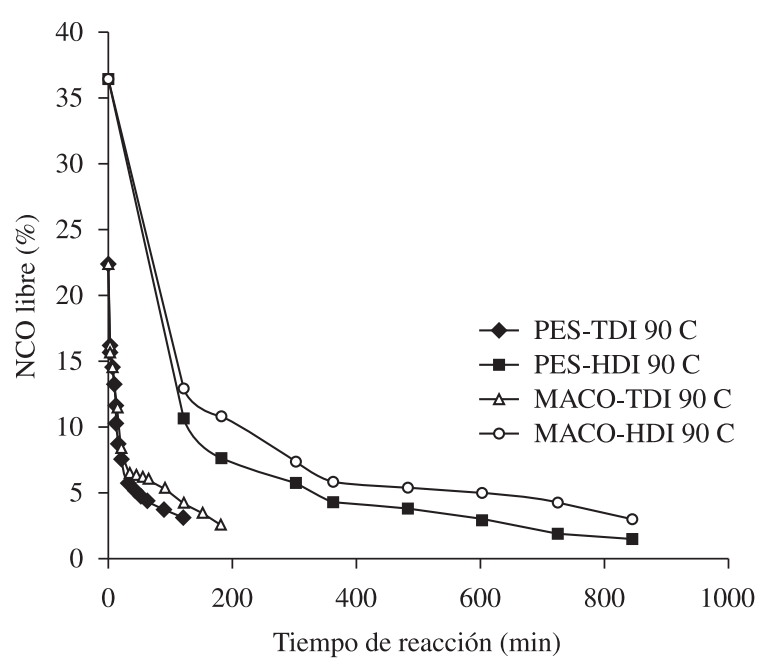

Figura 1. Variación del \%NCO libre para los sistemas PES FL-60-TDI, PES FL-60-HDI, MACO-TDI, MACO-HDI, con relación molar NCO:OH = 1, a $90^{\circ} \mathrm{C}$, sin catalizador.

La reactividad del TDI es mayor que el HDI, la constante de velocidad posee un valor superior, esto es debido al carácter aromático de TDI que hace que se facilite el ataque 


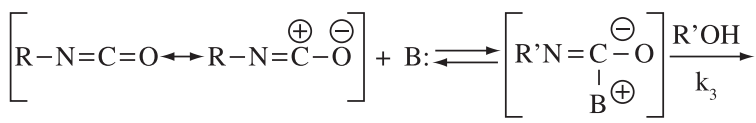

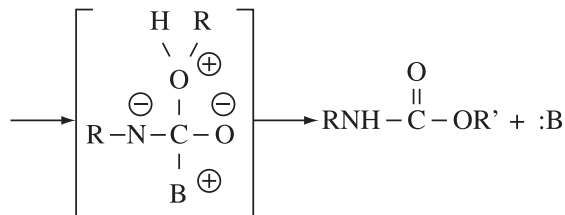

Figura 2. Mecanismo de la catálisis básica en la formación de PU.

Tabla 3. Valores de constante de velocidad para la reacción de poliester (PES FL-60) y MACO con TDI y HDI.

\begin{tabular}{lllc}
\hline & & $\mathbf{k}_{1}{ }^{*} \mathbf{1 0} \mathbf{1}^{-4}(\mathbf{L} / \mathbf{m o l}-\mathbf{s})$ & $\mathbf{k}_{2}{ }^{*} \mathbf{1 0}^{-4}(\mathbf{L} / \mathbf{m o l}-\mathbf{s})$ \\
& & $\mathbf{R}^{\mathbf{2}}$ & $\mathbf{R}^{\mathbf{2}}$ \\
\hline PES FL-60 & TDI & 6.571 & 2.308 \\
& & 0.9887 & 0.9973 \\
PES FL-60 & \multirow{2}{*}{ HDI } & 0.921 & ---- \\
& & 0.9327 & \\
MACO & \multirow{2}{*}{ TDI } & 5.151 & 1.984 \\
& & 0.9683 & 0.8986 \\
MACO & \multirow{2}{*}{ HDI } & 0.474 & ---- \\
& & 0.9502 & \\
\hline
\end{tabular}

nucleofílico al nitrógeno del grupo isocianato por el efecto inductivo de sacar electrones. El poliester (PES FL-60) reacciona más rápido que MACO, debido a sus hidroxilos primarios; para disminuir los tiempos de reacción se debe emplear un catalizador como octoato de estaño.

El porcentaje de NCO libre requerido para realizar una buena dispersión es de 3 a $5 \%$, el cual se obtiene en: 120 minutos para el sistema PES FL-60-TDI, 500 minutos para PES FL-60-HDI, 180 minutos para MACO-TDI y 720 minutos para MACO-HDI a $90{ }^{\circ} \mathrm{C}$.

Para facilitar la reacción y disminuir el tiempo de reacción, se debe adicionar $0,1 \mathrm{~g}$ de octoato de estaño, el cual es un catalizador organometálico.

\section{Dispersión por inversión de fases. Optimización}

El análisis estadístico se realiza empleando el software StatGraphics 5.1, empleando una ecuación polinomial de segundo orden (Ecuacion 3):

$$
z=\beta_{0}+\beta_{1} x+\beta_{2} y+\beta_{3} x^{2}+\beta_{4} y^{2}+\beta_{5} x y
$$

donde: $\mathrm{z}$ : variable de respuesta porcentaje de sedimentación; $\mathrm{x}$ : variable independiente codificada del porcentaje de ácido cítrico (\%cítrico); y: variable codificada correspondiente a la relación NCO:OH; $\beta_{0}, \beta_{1}, \beta_{2}, \beta_{3}, \beta_{4}, \beta_{5}$ son los coeficientes respectivos del intercepto, lineal, cuadrática e interacción.

Para determinar el punto estacionario se calcula la derivada de la superficie de respuesta con respecto a cada una de las variables codificadas y se resuelve el sistema de ecuaciones, luego se calculan los valores reales (Ecuacion 4).

$$
\frac{\partial Z}{\partial y}=0 \rightarrow y \frac{\partial Z}{\partial x}=0 \rightarrow x
$$

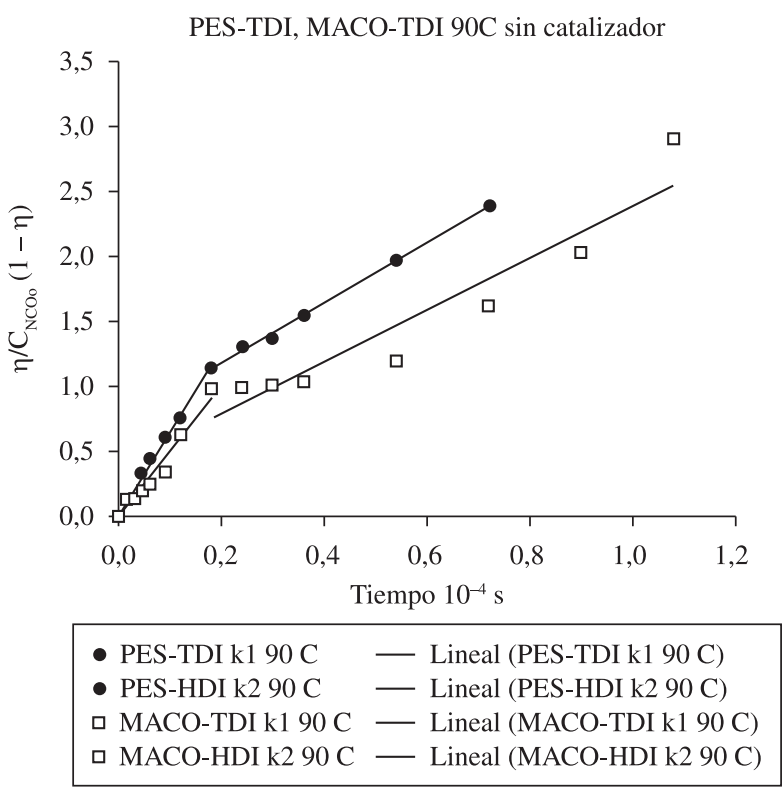

(a)

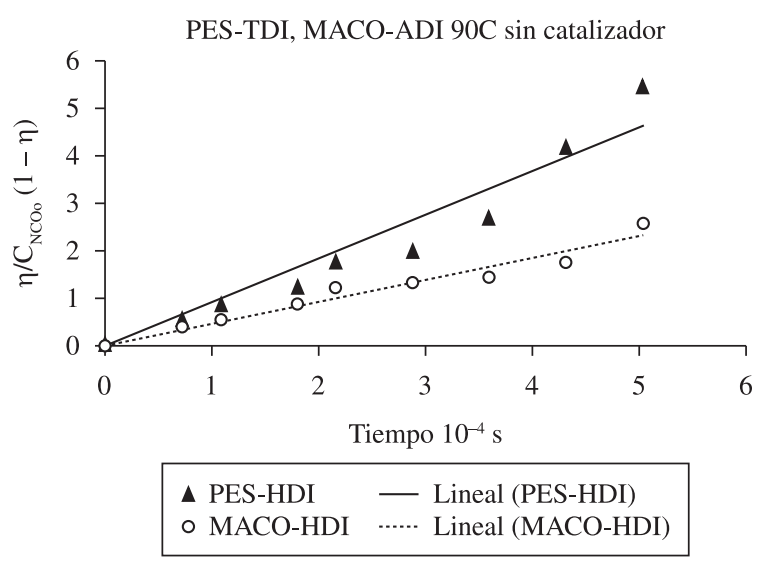

(b)

Figura 3. a) Cinética de segundo orden para la reacción PES FL-60-TDI y MACO-TDI, sin catalizador. b) Cinética de segundo orden para la reacción PES FL-60-HDI y MACO-HDI, sin catalizador.

Para determinar si el punto estacionario corresponde a un máximo, mínimo o punto silla, se debe emplear la matriz Hessiana, la cual es (Ecuacion 5):

$$
H=\left(\begin{array}{cc}
\frac{\partial^{2} z}{\partial^{2} x} & \frac{\partial^{2} z}{\partial x \partial y} \\
\frac{\partial^{2} z}{\partial y \partial x} & \frac{\partial^{2} z}{\partial^{2} y}
\end{array}\right)
$$

Y se calculan los determinantes de las submatrices (Det), dependiendo del signo obtenido podemos clasificar el punto estacionario así:

- Det $\mathrm{H}_{1}<0$ y Det $\mathrm{H}_{2}>0$, el punto es un máximo;

- Det $\mathrm{H}_{1}>0$ y Det $\mathrm{H}_{2}>0$, el punto es mínimo;

- en otros casos es silla.

Los valores experimentales obtenidos para el porcentaje de sedimentación evaluado en las muestras después de 1 mes 
de elaboradas las dispersiones con $40 \%$ en sólidos se reporta en la Tabla 4.

Se realizó el análisis de estos parámetros con el estadístico t para cada uno de los sistemas de reacción como se ilustra en la Tabla 5, los p-valores mayores que 0.05 , indican que no hay relaciones estadísticamente significativas entre las variables a un nivel de confianza del 95\%, pero en este análisis de regresión múltiple se consideran todos los coeficientes.

Los términos que no son significativos de acuerdo a la prueba t se resaltan en la Tabla 5, pero para la optimización se utilizan todos los términos. También se aprecia en el análisis de varianza que el modelo de regresión es significativo, y de acuerdo a los coeficientes de determinación, $\mathrm{R}^{2}$ y $\mathrm{Raj}^{2}$, el modelo explica bien la variabilidad presente en los datos. También se aprecia el error estándar de estimación (s) que dada la escala de medición de la variable de respuesta, éstos tienen una magnitud relativamente pequeña, sin embargo puede generarse error en el análisis estadístico debido a que los niveles de la concentración de ácido cítrico no son equidistantes en el punto central.
Los puntos estacionarios obtenidos corresponden con un mínimo, y sus valores se muestran en la Tabla 6.

Los sistemas obtenidos con MACO presentan el menor porcentaje de sedimentos en las dispersiones obtenidas, esto es debido a que los grupos ácido que no alcanzan a reaccionar dentro de la estructura del triglicérido, se neutralizan y pueden ejercer un efecto emulsificante en las muestras, por otro lado, la mayor reactividad del TDI y su menor polaridad con respecto al HDI, hacen que este interactúe más fácilmente con el aceite y se produzca mejor miscibilidad, lo podría explicar la mayor estabilidad.

\section{Caracterización de los PUD'S}

Los adhesivos fueron sintetizados de acuerdo a los puntos estacionarios obtenidos después de la optimización y se caracterizan de acuerdo con las normas ASTM enunciadas anteriormente, los resultados se muestran en la Tabla 7.

Para la clasificación de adhesión se determina: baja en un rango de 0-500, media de 500-1000 y alta entre 1000-1500 gf, obtenidas de la prueba de Peel. Para la cohesión: 0-40 baja, 40-80 media y > 80 minutos alta, para la prueba de Hold.

Tabla 4. Porcentaje de sedimentación de las dispersiones para los diferentes sistemas.

\begin{tabular}{|c|c|c|c|c|c|c|}
\hline & \% CITRICO & NCO:OH & $\%$ sed PES-TDI & $\%$ sed PES-HDI & $\%$ sed MACO-TDI & \% sed MACO-HDI \\
\hline 1 & -1 & -1 & 14.33 & 21.86 & 4.45 & 25.63 \\
\hline 2 & 1 & -1 & 5.53 & 41.45 & 1.09 & 47.74 \\
\hline 3 & 0 & 1 & 5.68 & 0.49 & 4.07 & 1.37 \\
\hline 4 & 1 & 0 & 0.90 & 48.52 & 3.46 & 50.52 \\
\hline 5 & 0 & -1 & 14.68 & 1.93 & 5.08 & 1.72 \\
\hline 6 & 1 & 1 & 5.54 & 57.98 & 5.09 & 40.75 \\
\hline 7 & 0 & 0 & 0.19 & 0.94 & 0.58 & 2.03 \\
\hline 8 & -1 & 1 & 30.10 & 15.74 & 12.57 & 13.78 \\
\hline 9 & -1 & 0 & 21.75 & 9.37 & 9.93 & 7.12 \\
\hline 10 & 0 & 0 & 1.01 & 0.0075 & 0.90 & 0.17 \\
\hline 11 & 0 & 0 & 0.63 & 1.75 & 1.83 & 0.57 \\
\hline 12 & 0 & 0 & 1.35 & 2.49 & 1.76 & 2.49 \\
\hline 13 & 0 & 0 & 2.74 & 1.87 & 2.84 & 3.87 \\
\hline 14 & 0 & 0 & 4.99 & 3.12 & 0.85 & 2.79 \\
\hline
\end{tabular}

Tabla 5. Estimación de los coeficientes de regresión de \% sólidos sedimentados (z).

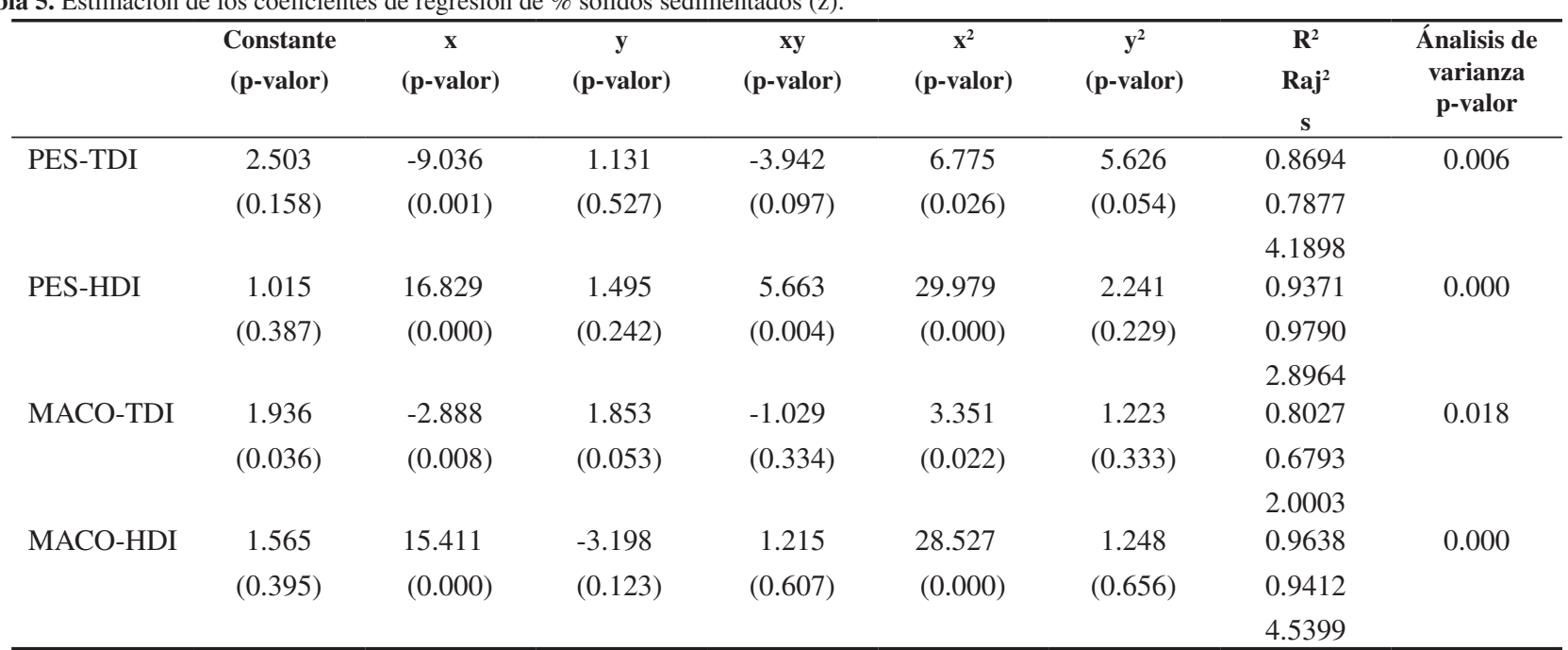

Donde S: desviación estándar, Raj $^{2}$ = ajustada. 
Tabla 6. Puntos estacionarios obtenidos.

\begin{tabular}{|c|c|c|c|c|c|}
\hline & \multicolumn{2}{|c|}{ Valores codificados } & \multicolumn{2}{|c|}{ Valores reales } & \multirow{2}{*}{$\begin{array}{l}\text { Naturaleza puntc } \\
\text { estacionario }\end{array}$} \\
\hline & $\mathbf{x}$ & $\mathbf{y}$ & \% Cítrico & $\mathrm{NCO} / \mathrm{OH}$ & \\
\hline PES-TDI & 0.7099 & 0.1482 & 0.6630 & 1.1300 & Mínimo \\
\hline PES-HDI & -0.2829 & 2.3939 & 0.5349 & 1.5800 & Mínimo \\
\hline MACO-TDI & 0.3363 & -0.6161 & 0.5509 & 0.9768 & Mínimo \\
\hline MACO-HDI & 0.3005 & 1.4271 & 0.5401 & 1.3854 & Mínimo \\
\hline
\end{tabular}

Tabla 7. Resultados pruebas de caracterización.

\begin{tabular}{|c|c|c|c|c|c|c|c|}
\hline Muestra & Película & $\begin{array}{c}\text { Fuerza adhesiva } \\
\text { (Peel) } \\
\text { [gf] }\end{array}$ & $\begin{array}{c}\text { Depósito } \\
{\left[\text { g.m }{ }^{-2}\right]}\end{array}$ & $\begin{array}{c}\text { Fuerza } \\
\text { cohesiva } \\
(\text { Hold }) \\
{[\text { min] }}\end{array}$ & $\begin{array}{c}\text { Loop tack } \\
{[\text { [lbf] }}\end{array}$ & $\begin{array}{l}\text { Tack } \\
{[\mathrm{cm}]}\end{array}$ & $\begin{array}{c}\mathrm{Tg} \\
{\left[{ }^{\circ} \mathbf{C}\right]}\end{array}$ \\
\hline MACO-TDI & - Uniforme & $1926.89 \pm 121.79$ & 17.35 & 311.6 & 2.52 & $>25$ & -3 \\
\hline MACO-HDI & $\begin{array}{l}\text { - Uniforme } \\
\text { - Brillante } \\
\text { - Presencia de } \\
\text { cráteres }\end{array}$ & $1578.26 \pm 37.44$ & 17.35 & 54.0 & 4.64 & 14.7 & -10 \\
\hline PES-TDI & $\begin{array}{l}\text { - Se abre al } \\
\text { aplicarla }\end{array}$ & $29.22 \pm 2.80$ & 17.35 & N/A & 0.11 & $>25$ & -30 \\
\hline PES-HDI & - Uniforme & $688.86 \pm 17.06$ & 17.35 & 9.1 & 2.56 & 6.9 & -47 \\
\hline $\begin{array}{l}\text { Autoadhesivo } \\
\text { acrílico }\end{array}$ & - Uniforme & $1280-1640$ & $16-20$ & $>100$ & $>2.3$ & $<6$ & -60 \\
\hline
\end{tabular}
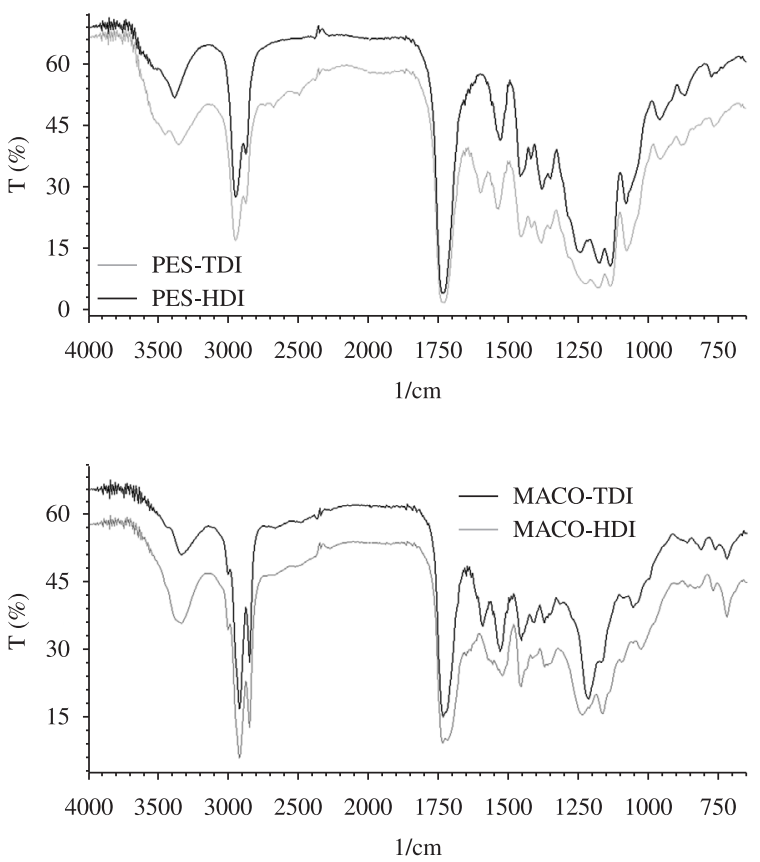

Figura 4. Espectros muestras PES-TDI y PES-HDI, Espectros muestras MACO-TDI y MACO-HDI.

Las muestras obtenidas con MACO presentaron una buena adhesión y cohesión, película uniforme y un valor de loop tack superior a los adhesivos acrílicos y SBR (Tabla 7).

Las muestras obtenidas con PES FL - 60 poseen mala adhesión y cohesión, un valor de loop tack bajo con respecto a los acrílicos y la película tiende a abrirse en el momento de aplicación debido a una alta tensión superficial.

Los adhesivos reposicionables poseen una $\mathrm{Tg}$ baja (Tabla 7), lo que garantiza su flexibilidad, sin embargo, las dispersiones realizadas con TDI tienden a tener en su estructura cadenas rígidas que hace que poseean una $\mathrm{Tg}$ más alta. Hay que tener en cuenta que a mayor cantidad de diisocianato se genera mayor rigidez.

Como los poliésteres son macrodioles y MACO es un triol, el entrecruzamiento de este último es mucho más alto, por lo que el $\mathrm{Tg}$ de las muestras sintetizadas con éste tiende a ser más alto.

La Figura 4 a y b muestran los espectros IR obtenidos de los adhesivos obtenidos

Los espectros IR de las muestras muestran señales a $3380 \mathrm{~cm}^{-1}$ que se deben a la tensión O-H de los grupos hidroxilo libres y tensión N-H; a 2750-2950 cm-1 aparecen señales debidas a la tensión $\mathrm{C}-\mathrm{H}$ de las cadenas hidrocarbonadas; la señal a $1680-1750 \mathrm{~cm}^{-1}$ se debe a estiramientos $\mathrm{C}=\mathrm{O}$ de los grupos ester que se superpone también a la de estiramientos $\mathrm{C}=\mathrm{O}$ de los grupos uretano, urea y amida.

En los espectros de las dispersiones obtenidas a partir de TDI, aparece también una señal a $1600 \mathrm{~cm}^{-1}$, típica de anillos aromáticos provenientes de dicho isocianato.

En $2270 \mathrm{~cm}^{-1}$ no aparece la señal de los grupos R-NCO, ya que este reacciona en su totalidad con cualquier grupo reactivo en el sistema.

\section{Conclusiones}

Se obtuvieron dispersiones acuosas de poliuretano con buenas propiedades físicas y mecánicas empleando un poliol de fuentes renovables (MACO), para reemplazar a los polioles comerciales. El ácido cítrico como agente emulsificante 
interno, permitió obtener un producto económico y de alta durabilidad, empleando materias primas de menor costo y de fácil consecución, sin alterar significativamente las propiedades y características de las dispersiones que se producen comercialmente en la actualidad. Igualmente se logró cambiar el IPDI, comúnmente empleado en dispersiones acuosas, por otros isocianatos como TDI y HDI.

\section{Agradecimientos}

Los autores agradecen al Programa Estrategia de Sostenibilidad de Grupos de la Universidad de Antioquia.

\section{References}

1. Woods, G.W. “The ICI polyurethanes book”, John-Wiley, Netherlands (1987).

2. Carme, M.; Babb, D. \& Ryan, A. -Polymer, 49, p.3279-3287 (2008).

3. Szycher, M. "Szycher's Handbook of Polyurethanes", CRC Press, Boca Raton, Florida (1999).

4. Urban, D. \& Takamura, K. "Polymer Dispersions and Their Applications", Wiley-VCH, Germany (2002).
5. Jang, J. Y.; Jhon, Y. K.; Cheong, I. W. \& Kim, J. H. Physicochem. Eng. Asp., 196, p.135-143 (2002).

6. Ionescu, M. "Chemistry and technology of polyols for polyurethanes", Rapra Technology Limited, Shawbury (2005).

7. Costa, M.; Ramos, V.; Abrantes, T.; Castro, D.; Visconte, L.; Nunes, R. \& Furtado, C. - Polímeros, 14, 1, p.46-50 (2004).

8. Cangemi, J.; Neto, S.; Chierice, G. \& Dos Santos, A. Polímeros, 16, 2, p.129-135 (2006).

9. Cangemi, J.; Dos Santos, A.; Neto, S. \& Chierice, G. Polímeros, 18, 3, p.201-206 (2008).

10. Mazo, P.; Rios, L.; \& Estenoz, D. - Polímeros, 19, 2, p.149-154 (2009).

11. Saunders, J. \& Frisch, K. C. "Polyurethane. Chemistry and Technology, Part I", Wiley- Interscience, New York (1962).

12. Ajithkumar, S.; Kansara, S. \& Patel, N. - Eur. Polym. J., 34, 9, p.1273-1276 (1998).

Enviado: 21/09/09

Reenviado: $30 / 12 / 09$

Aceito: $29 / 01 / 10$

DOI: 10.1590/S0104-14282010005000022 